\section{(1) \\ CrossMark}

\title{
Role of common hypnotics on the phenotypic causes of obstructive sleep apnoea: paradoxical effects of zolpidem
}

\author{
Jayne C. Carberry ${ }^{1}$, Lauren P. Fisher ${ }^{1}$, Ronald R. Grunstein ${ }^{2}$, \\ Simon C. Gandevia ${ }^{1,3}$, David K. McKenzie ${ }^{3}$, Jane E. Butler ${ }^{1}$ and Danny J. Eckert ${ }^{1}$ \\ Affiliations: ${ }^{1}$ Neuroscience Research Australia, Sydney, Australia. ${ }^{2}$ The Woolcock Institute of Medical \\ Research, Sydney, Australia. ${ }^{3}$ Prince of Wales Hospital, Sydney, Australia. \\ Correspondence: Jayne C. Carberry, Neuroscience Research Australia PO Box 1165, Randwick, NSW, 2031, \\ Australia. E-mail: j.carberrylaneura.edu.au
}

@ERSpublications

Contrary to previous beliefs, common sleeping pills do not reduce pharyngeal muscle activity in humans http://ow.ly/f69H30gKIot

Cite this article as: Carberry JC, Fisher LP, Grunstein RR, et al. Role of common hypnotics on the phenotypic causes of obstructive sleep apnoea: paradoxical effects of zolpidem. Eur Respir J 2017; 50: 1701344 [https://doi.org/10.1183/13993003.01344-2017].

ABSTRACT Hypnotics are contraindicated in obstructive sleep apnoea (OSA) because of concerns of pharyngeal muscle relaxation and delayed arousal worsening hypoxaemia. However, human data are lacking. This study aimed to determine the effects of three common hypnotics on the respiratory arousal threshold, genioglossus muscle responsiveness and upper airway collapsibility during sleep.

21 individuals with and without OSA (18-65 years) completed 84 detailed sleep studies after receiving temazepam $(10 \mathrm{mg})$, zolpidem $(10 \mathrm{mg})$, zopiclone $(7.5 \mathrm{mg})$ and placebo on four occasions in a randomised, double-blind, placebo-controlled, crossover trial (ACTRN12612001004853).

The arousal threshold increased with zolpidem and zopiclone versus placebo (mean $\pm \mathrm{SD}-18.3 \pm 10$ and $-19.1 \pm 9$ versus $-14.6 \pm 7 \mathrm{cmH}_{2} \mathrm{O} ; \mathrm{p}=0.02$ and $\left.\mathrm{p}<0.001\right)$ but not with temazepam $\left(-16.8 \pm 9 \mathrm{cmH}_{2} \mathrm{O}\right.$; $\mathrm{p}=0.17)$. Genioglossus muscle activity during stable non-REM sleep and responsiveness during airway narrowing was not different with temazepam and zopiclone versus placebo but, paradoxically, zolpidem increased median muscle responsiveness three-fold during airway narrowing (median -0.15 (interquartile range -1.01 to -0.04$)$ versus $-0.05(-0.29$ to -0.03$) \%$ maximum EMG per $\mathrm{cmH}_{2} \mathrm{O}$ epiglottic pressure; $\mathrm{p}=0.03$ ). The upper airway critical closing pressure did not change with any of the hypnotics.

These doses of common hypnotics have differential effects on the respiratory arousal threshold but do not reduce upper airway muscle activity or alter airway collapsibility during sleep. Rather, muscle activity increases during airway narrowing with zolpidem.

This article has supplementary material available from erj.ersjournals.com

Received: July 052017 | Accepted after revision: Sept 262017

This study was registered at the Australian New Zealand Clinical Trial Registry as ACTRN12612001004853.

Support statement: This study was funded by a National Health and Medical Research Council (NHMRC) of Australia project grant (1042493) and NeuroSleep, a NHMRC Centre for Research Excellence (1060992). D.J. Eckert was supported by a NHMRC RD Wright Fellowship (1049814) and is currently supported by a NHMRC Senior Research Fellowship (1116942). R.R. Grunstein, J.E. Butler and S.C. Gandevia are also supported by NHMRC Fellowships. Funding information for this article has been deposited with the Crossref Funder Registry.

Conflict of interest: Disclosures can be found alongside this article at erj.ersjournals.com

Copyright CERS 2017 


\section{Introduction}

The use of hypnotics is widespread. More than $5 \%$ of the general population report hypnotic use within the past month [1]. Usage rates are 1.5-2 times higher in older people (>60 years) and in those who are obese; two major risk factors for obstructive sleep apnoea (OSA) [1-3]. Hypnotics act as central nervous system depressants (e.g. benzodiazepines) and have traditionally been discouraged in people with OSA. Primary concerns have been that hypnotics reduce upper airway muscle activity and delay arousal from sleep, leading to worsening of upper airway stability and increased respiratory event duration and hypoxaemia [4]. However, evidence to support these concerns is limited.

An early study indicated that increases in genioglossus muscle activity to $\mathrm{CO}_{2}$ rebreathing during wakefulness are reduced in some people after receiving the benzodiazepine diazepam [5]. In contrast, recent studies have shown that standard doses of the non-benzodiazepine hypnotic zopiclone do not reduce upper airway muscle activity during sleep $[6,7]$ nor does the tetracyclic antidepressant trazodone [8]. Standard doses of common hypnotics also increase the threshold for arousal from sleep by $20-50 \%$ [9]. High doses of certain hypnotics in people with severe OSA can worsen hypoxaemia [6, 10, 11]. However, other studies show either no change in hypoxaemia or the apnoea-hypopnoea index (AHI) [12$14]$. Given that arousals from sleep can be deleterious for many OSA patients $[9,15]$, certain hypnotics reduce the AHI $[16,17]$, particularly in those who wake easily to airway narrowing (i.e. have a low respiratory arousal threshold [17]). $30-50 \%$ of OSA patients [16-18] and up to $\sim 85 \%$ in some patient groups (e.g. non-obese individuals) [19] have a low respiratory arousal threshold. Thus, some hypnotics may promote breathing stability for certain people with OSA. However, if hypnotics reduce upper airway muscle activity and worsen airway collapsibility, this could limit any benefit from suppression of arousal.

Given continuing uncertainty about the actions of hypnotics on upper airway function and breathing, this study aimed to systematically determine the effects of three commonly used hypnotics (temazepam, zolpidem and zopiclone) on key phenotypic contributors to OSA pathogenesis including 1) the respiratory arousal threshold, 2) upper airway dilator muscle activity, 3) upper airway collapsibility and 4) breathing during sleep in people with and without OSA.

\section{Methods}

Subjects

28 men and women were recruited by advertisement to participate and provided written informed consent. The study was approved by the University of New South Wales Human Research Ethics Committee (HC12028). Participants with (>10 events.h ${ }^{-1}$ sleep) and without OSA were otherwise healthy and not taking any medications known to effect sleep or breathing.

\section{Measurements and equipment \\ Polysomnography}

Electroencephalograms (EEGs), electro-oculograms (EOGs) and submentalis electromyograms (EMGs) were used to determine sleep stage and cortical arousals according to standard criteria [20].

\section{Physiological measurements}

A pressure-tipped catheter (Millar, TX, USA) was inserted into a decongested (phenylephrine hydrochloride, Nyal; iNova Pharmaceuticals (Australia), Chatswood, Australia) and anaesthetised nostril (lignocaine, Co-phenylcaine Forte $5 \%$ ) and advanced $\sim 1.5-2 \mathrm{~cm}$ below the base of the tongue to determine the epiglottic pressure. Following surface anaesthesia (1\% lignocaine), two stainless-steel Teflon-coated intramuscular electrodes (no. 791500; A-M Systems Inc., Sequim, WA, USA) with $\sim 1.5 \mathrm{~mm}$ of insulation removed were inserted orally using a 25 -gauge needle $\sim 4 \mathrm{~mm}$ either side of the frenulum 15$20 \mathrm{~mm}$ deep, to measure genioglossus EMG in a bipolar configuration as described previously [21]. Participants were fitted with a nasal mask attached to a heated pneumotachograph (model 3700A; Hans Rudolph Inc., Shawnee, KS, USA) and differential pressure transducers to measure airflow and mask pressure $\left(P_{\text {mask }}\right)$.

\section{Protocol}

Participants were randomised to a sequence to receive either temazepam (10 mg), zolpidem (10 mg), zopiclone $(7.5 \mathrm{mg})$ or placebo on four separate nights just prior to sleep, with 1 week wash-out between visits in a crossover trial (figure 1). After instrumentation, the maximal genioglossus EMG was determined from large swallows or tongue protrusions [22]. A period of quiet breathing during wakefulness was acquired to quantify baseline genioglossus EMG and breathing. Continuous positive airway pressure (CPAP) was applied at a holding pressure (i.e. therapeutic CPAP level for people with OSA or $\sim 4 \mathrm{cmH}_{2} \mathrm{O}$ for non-OSA participants). After receiving the study intervention, participants were instructed to sleep supine. Once stable sleep was achieved, CPAP was reduced repeatedly throughout the night to varying 


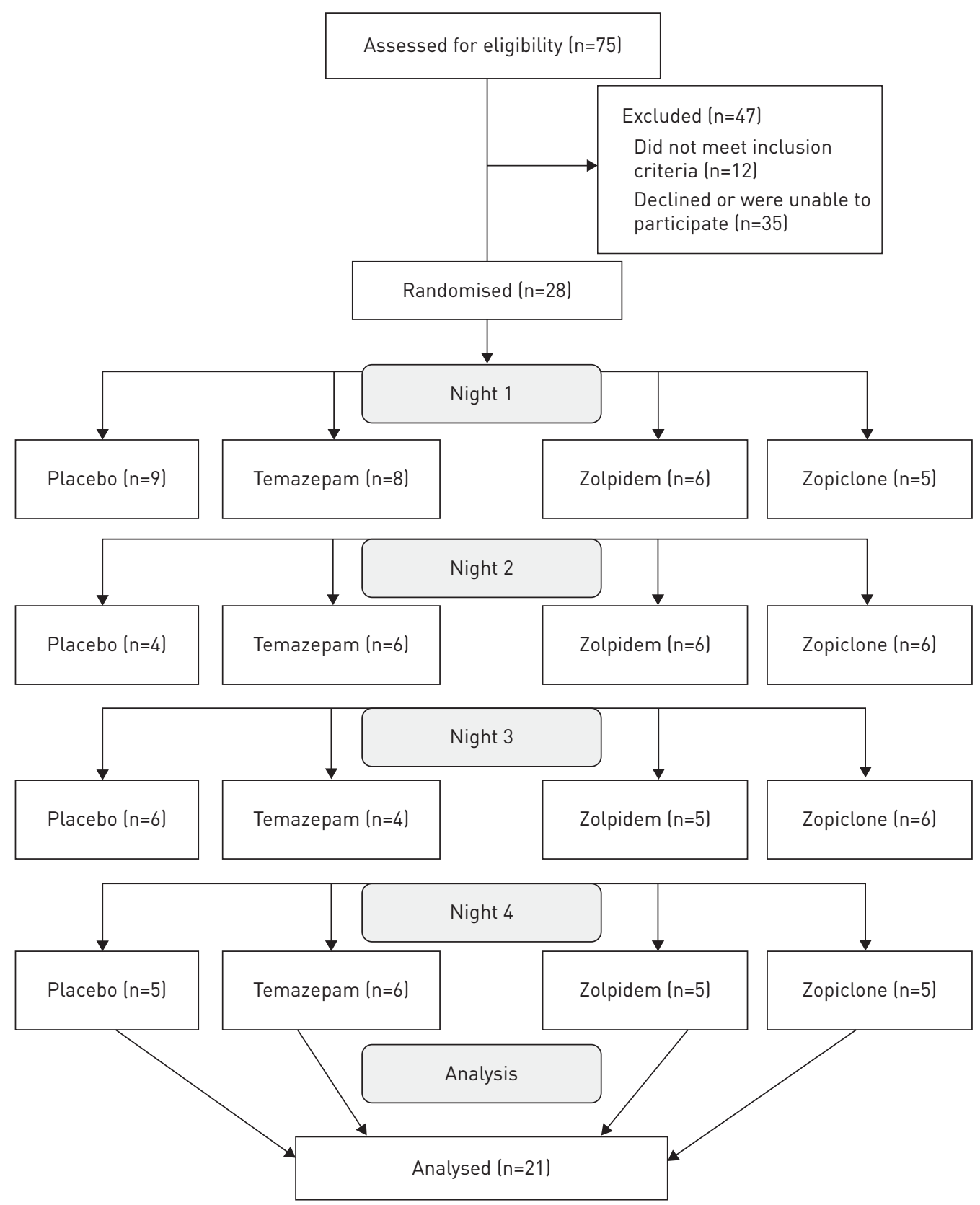

FIGURE 1 CONSORT diagram illustrating the participant recruitment, randomisation and analysis flow for this double-blind, placebo-controlled, cross-over study. $n=12$ participants were excluded from enrolment based on their age (>65 years), medication use or smoking history. Participants with obstructive sleep apnoea had an apnoea-hypopnea index greater than 10 events $\cdot h^{-1}$. Participants were required to complete four overnight sleep studies. $n=7$ participants withdrew from the study prior to completion of all four nights. $n=21$ participants completed all four nights.

pressures below the holding level, for up to $3 \mathrm{~min}$ to induce airway narrowing or collapse to allow for quantification of the phenotypic traits (figure 2) [21]. The modified CPAP device (Philips Pcrit3000, PA, USA) also enabled suction pressures to ensure airway narrowing in all participants.

\section{Data acquisition and analysis}

Data were acquired using an analogue-to-digital convertor (1401) with Spike2 software (Cambridge Electronic Design Ltd, Cambridge, UK). EMG signals were amplified (CED 1902) and sampled at $1000 \mathrm{~Hz}$ (band-pass 30-500 Hz). EOG, EEG and other signals were sampled at $250 \mathrm{~Hz}$.

The genioglossus EMG was rectified, averaged (100 ms moving-time average) and expressed as a percentage of the maximal activity achieved from the tongue protrusions or swallows. The respiratory 


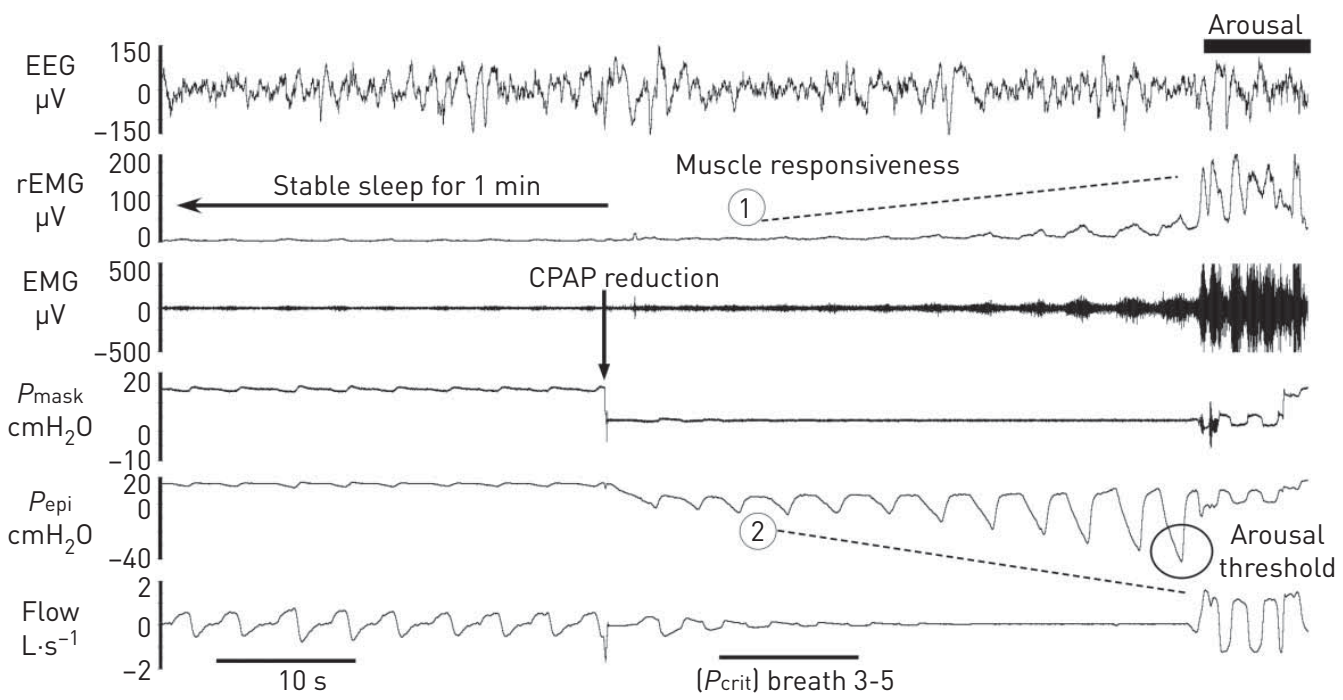

FIGURE 2 Example of a continuous positive airway pressure (CPAP) reduction during stable non-REM sleep showing narrowing (hypopnoea) and closure (apnoea) of the upper airway. Multiple CPAP drops were applied throughout the night. Genioglossus electromyography (EMG) activity and breathing parameters were determined for $1 \mathrm{~min}$ prior to each CPAP reduction. Arousal threshold was determined as the nadir epiglottic pressure $\left(P_{\text {epi }}\right)$ immediately preceding cortical arousal (black bar). Muscle responsiveness was determined as the slope of peak genioglossus muscle activity (1) versus increasing negative epiglottic pressure (2). Upper airway collapsibility measured via the passive critical closing pressure $\left(P_{\text {crit }}\right)$ technique was determined from flow-limited breaths 3-5 following the reduction in CPAP. $P_{\text {mask }}$ : mask pressure; rEMG: rectified moving-time averaged EMG; EEG: electroencephalogram. Note: the raw EMG signal is clipped during the arousal.

arousal threshold, genioglossus muscle responsiveness and passive critical closing pressure $\left(P_{\text {crit }}\right)$ were quantified as shown in Figure 2 and described previously [21].

\section{Statistical procedures}

Statistical analyses were performed blinded using SPSS and SigmaPlot (version 24; IBM, NY, USA, and version 12, Systat software Inc. USA, respectively). Our a priori statistical approach was to compare the effect of each hypnotic versus placebo for each key outcome where data were obtained during all four nights using paired t-tests or Wilcoxon signed rank tests for non-parametric data. p-values were adjusted for multiple comparisons using the Dunn-Sidák method [23]. Based on previous hypnotic studies [8, 17], this study was designed to detect a $4 \mathrm{cmH}_{2} \mathrm{O}$ difference (between night standard deviation 4.6) for the primary outcome (respiratory arousal threshold) between each hypnotic versus placebo based on a sample size of 20 with $>80 \%$ power.

The goal of this within-subjects crossover study was to examine the effects of common hypnotics on the phenotypic traits that cause OSA versus placebo in people with and without OSA rather than to compare differential effects between groups. Nonetheless, potential interaction effects (group-by-drug) were explored using two-way repeated measures ANOVA or mixed model analyses as appropriate. Exploratory one-way repeated measures ANOVA were also used to compare the effect of drug on the primary outcome (arousal threshold).

Permutation tests using program $\mathrm{R}$ (version 3.4, Vienna, Austria) were used to compare potential differences in variance of changes in genioglossus muscle responsiveness with each of the study drugs versus placebo. Adjusted $\mathrm{p}$-values $<0.05$ were considered statistically significant. Confidence intervals were also reported for the key outcomes.

\section{Results}

\section{Subject and stimulus characteristics}

In accordance with an anticipated dropout rate of $20-30 \%$ for this complex four-night study, 28 participants were recruited and 21 completed the four-night protocol (three withdrew prior to data collection on night 1 following instrumentation, and four completed fewer than four nights). This includes complete data in 20 participants for the primary outcome (target sample size). Anthropometric characteristics for the 21 participants ( $\mathrm{n}=10$ patients with OSA, mean \pm SD AHI $34 \pm 20$ events. $\mathrm{h}^{-1}$ ) who completed the study are shown in table 1 . 
TABLE 1 Anthropometric characteristics

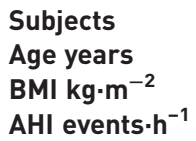

21 (7 females)

$40 \pm 15$

$28 \pm 7$

$19 \pm 22(0-77)$

Data are presented as $\mathrm{n}$, mean \pm SD (with range as appropriate). $\mathrm{n}=10$ had obstructive sleep apnoea. BMI: body mass index; $\mathrm{AHI}$ : apnoea-hypopnea index.

The total mean \pm SD number of CPAP reductions delivered during sleep per subject was $25 \pm 9,27 \pm 6,26 \pm 5$ and $29 \pm 5$ for placebo, temazepam, zolpidem and zopiclone respectively.

$11 \pm 6,9 \pm 4,9 \pm 5$ and $9 \pm 4$ CPAP reductions per condition were excluded from analysis because of immediate arousal (within $10 \mathrm{~s}$ ) or poor signal quality. Thus, $14 \pm 6,16 \pm 6,17 \pm 5$ and $19 \pm 5$ CPAP reductions for placebo, temazepam, zolpidem and zopiclone respectively were used to quantify the phenotypic traits during non-REM sleep.

\section{OSA versus non-OSA participants}

There were no group-by-drug interaction effects for any of the key study outcomes (e.g. arousal threshold $(\mathrm{p}=0.07)$, genioglossus muscle activity $(\mathrm{p}=0.348)$ and responsiveness $(\mathrm{p}=0.095), P_{\text {crit }}(\mathrm{p}=0.85)$ or breathing (minute ventilation; $\mathrm{p}=0.32)$ ). Thus, data in people with and without OSA were combined for all remaining analyses.

\section{Breathing and genioglossus EMG activity during stable sleep on CPAP}

Ventilatory parameters and genioglossus EMG activity during stable non-REM sleep while on CPAP are shown in table 2. Minute ventilation, inspiratory time, expiratory time, peak inspiratory flow and upper airway resistance were not significantly different from placebo following temazepam, zolpidem and zopiclone ( $\mathrm{p}>0.05$, table 2$)$.

Breathing frequency increased $\left(<1\right.$ breath $\left.\cdot \mathrm{min}^{-1}\right)$ with all three drugs compared with placebo $(p \leqslant 0.03$, table 2). Tonic and peak genioglossus EMG activity during stable non-REM sleep on CPAP expressed in microvolts and as a percentage of maximal activity did not change with hypnotic administration (table 2).

Peak genioglossus EMG (median (interquartile range) 0.93 (0.66-1.78), 1.11 (0.376-4.138), 1.34 (0.53$2.19)$ versus $0.59(0.49-2.52) \%$ maximum, $\mathrm{p}=0.98$ and tonic EMG $(0.25(0.14-0.41), 0.29(0.19-0.48), 0.27$ (0.20-0.78) versus $0.30(0.12-0.45) \%$ maximum, $\mathrm{p}=0.86$ during REM sleep on CPAP was also not different following hypnotics (temazepam, zolpidem, zopiclone) compared to placebo $(n=15)$.

TABLE 2 Breathing parameters and genioglossus electromyogram (EMG) activity during stable non-REM sleep on continuous positive airway pressure (CPAP)

$\begin{array}{lll}\text { Placebo } & \text { Temazepam } & \text { Zolpidem }\end{array}$

\section{Breathing parameters $(n=21)$}

CPAP holding pressure

Minute ventilation $\mathrm{L} \cdot \mathrm{min}^{-1}$

Inspiratory time s

Expiratory time $\mathrm{s}$

Breath frequency breaths $\cdot \mathrm{min}^{-1}$

Peak inspiratory flow $\mathrm{L} \cdot \mathrm{S}^{-1}$

Rua $_{200} \mathrm{cmH}_{2} \mathrm{O} \cdot \mathrm{L}^{-1} \cdot \mathrm{s}^{-1}$

Genioglossus EMG ( $n=20)$

Tonic $\mu \mathrm{V}$

Tonic EMG \% max

Peak $\mu \mathrm{V}$

Peak EMG \% max
Temazepam

$\begin{array}{cc}7.6 \pm 3.6 & 6.9 \pm 3.2 \\ 6.3 \pm 1.2 & 6.0 \pm 1.7 \\ 1.68 \pm 0.22 & 1.67 \pm 0.23 \\ 2.58 \pm 0.48 & 2.55 \pm 0.61 \\ 14.6 \pm 2.1 & 14.9 \pm 2.4^{*} \\ 0.55 \pm 0.12 & 0.52 \pm 0.14 \\ 1.66 \pm 0.65 & 2.19 \pm 1.34\end{array}$

$1.87(1.27-2.71)$

$0.44(0.23-0.84)$

$4.34(1.9-9.44)$

$0.86(0.52-2.35)$

$2.35(0.87-6.66)$

$0.66(0.31-1.11)$

$4.59(1.59-17.30)$

$1.5(0.56-4.24)$

\section{$7.6 \pm 3.1$}

$6.3 \pm 1.7$

$1.64 \pm 0.25$

$2.50 \pm 0.53$

$15.1 \pm 2.5^{*}$

$0.56 \pm 0.16$

$1.60 \pm 0.59$

$3.12(0.97-6.70)$
$0.53(0.26-1.16)$
$5.42(2.19-26.78)$
$1.04(0.47-5.44)$
$7.8 \pm 3.3$

$6.7 \pm 1.7$

$1.64 \pm 0.27$

$2.45 \pm 0.52$

$15.4 \pm 2.7 *$

$0.55 \pm 0.11$

$1.86 \pm 1.06$

3.29 (2.50 to 5.60$)$ 0.84 (0.52 to 1.77 )

7.11 (4.33 to 13.97$)$

1.88 (1.06 to 2.82 )

Data are presented as mean \pm SD or median (interquartile range). Rua 200 : upper airway resistance measured at a flow rate of $200 \mathrm{~mL} \cdot \mathrm{s}^{-1}$.

*: significant difference compared to placebo. Refer to text for further detail. 


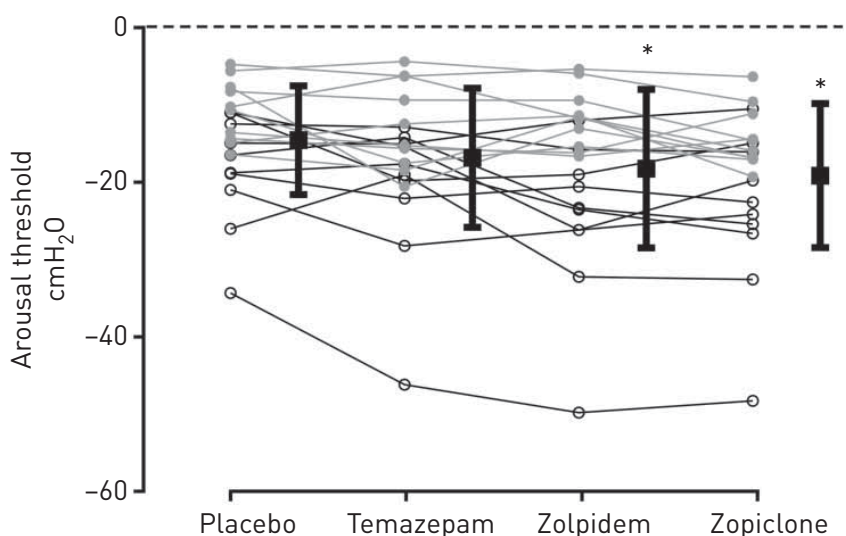

FIGURE 3 Scatterplot showing individual arousal thresholds for obstructive sleep apnoea (OSA) (open circles) and non-OSA participants (grey circles) during non-REM sleep for placebo and hypnotic conditions. Group mean $\pm S D$ are indicated with black squares adjacent to each condition. *: significant difference compared to placebo. Data in all four conditions were available in $n=20$ participants.

\section{Respiratory arousal threshold}

When compared with placebo, the mean \pm SD respiratory arousal threshold was $27 \pm 38 \%(\mathrm{p}=0.02)$ higher (i.e. harder to wake up) following zolpidem and $37 \pm 36 \%(\mathrm{p}<0.001)$ higher following zopiclone (figure 3 ). The respiratory arousal threshold with temazepam did not increase compared with placebo (19.9 $\pm 45.9 \%$ higher; $\mathrm{p}=0.17$ ). These findings were confirmed when expressed as the mean absolute difference between placebo and each drug (temazepam 2.3, 95\% CI -0.14 to 4.7 ; zolpidem 3.7, 95\% CI 1.3 to 6.2 ; and zopiclone $4.6,95 \%$ CI 2.5 to $6.6 \mathrm{cmH}_{2} \mathrm{O}$ ). Repeated measures ANOVA also revealed a significant main effect of drug on arousal threshold $(\mathrm{p}<0.001)$. The change in arousal threshold from placebo was not different between hypnotics $(\mathrm{p}=0.13)$. Time to arousal was not significantly different following temazepam, zolpidem or zopiclone $(42.9 \pm 16.7,42.3 \pm 22.5$ and $42.3 \pm 22.0$ versus placebo $41.5 \pm 15.2 \mathrm{~s} ; \mathrm{p}=0.98, \mathrm{p}=0.91, \mathrm{p}=0.99$ respectively).

\section{Genioglossus muscle responsiveness}

Genioglossus muscle responsiveness to airway narrowing and closure increased following zolpidem compared with placebo (figure 4$)(-0.15(-1.01$ to -0.04$)$ versus $-0.05(-0.29$ to -0.03$)) \%$ maximum genioglossus EMG per $\mathrm{cmH}_{2} \mathrm{O}$ epiglottic pressure (median and interquartile range; $\mathrm{p}=0.03$ ). In contrast, there was no difference in genioglossus muscle responsiveness with temazepam $(-0.12(-0.43$ to -0.05$))$ and zopiclone $(-0.07$ ( -0.31 to -0.04$)) \%$ maximum genioglossus $\mathrm{EMG} / \mathrm{cmH}_{2} \mathrm{O}$ epiglottic pressure compared to placebo ( $\mathrm{p}=0.54$ and $\mathrm{p}=0.98$, respectively) (figure 4 ). These findings were also confirmed when expressed as the mean difference between placebo and each drug and the $95 \%$ confidence intervals (figure 4 insets).

The variance in the change in genioglossus muscle responsiveness from placebo to temazepam was significantly greater than the variance of the change from placebo to zopilcone $(p=0.02)$. The variance for the other comparisons was similar (temazepam-zolpidem, $\mathrm{p}=0.12$ and zolpidem-zopiclone $\mathrm{p}=0.32$ ).

\section{Upper airway collapsibility}

Neither temazepam, zolpidem nor zopiclone changed passive upper airway collapsibility $\left(P_{\text {crit }}\right)$ during sleep compared with placebo $(\mathrm{p}=0.99, \mathrm{p}=0.98, \mathrm{p}=0.98$, respectively) (figure 5 ). These findings were confirmed when expressed as the mean difference between placebo and each drug (temazepam $0.004,95 \%$ CI -1.47 to 1.48 ; zolpidem 0.22 , $95 \%$ CI -1.25 to 1.7 ; and zopiclone $0.204,95 \%$ CI -1.23 to $\left.1.64 \mathrm{cmH}_{2} \mathrm{O}\right)$.

\section{Discussion}

The main findings of this study are that the non-benzodiazepine " $Z$ " drugs zolpidem (10 mg) and zopiclone $(7.5 \mathrm{mg}$ ) increase the respiratory arousal threshold by $27 \%$ and $37 \%$ respectively whereas $10 \mathrm{mg}$ of the benzodiazepine temazepam does not. The doses of all three of these hypnotics do not systematically reduce upper airway dilator muscle activity and do not impair minute ventilation during stable sleep while on CPAP. Genioglossus muscle responsiveness during airway narrowing is preserved with temazepam and zopiclone compared with placebo, but paradoxically, zolpidem increases muscle responsiveness. Finally, there is no evidence that upper airway collapsibility $\left(P_{\text {crit }}\right)$ during sleep is altered by these common hypnotics. These findings challenge previous assumptions [4] that hypnotics make OSA worse because of pharyngeal muscle relaxation. 

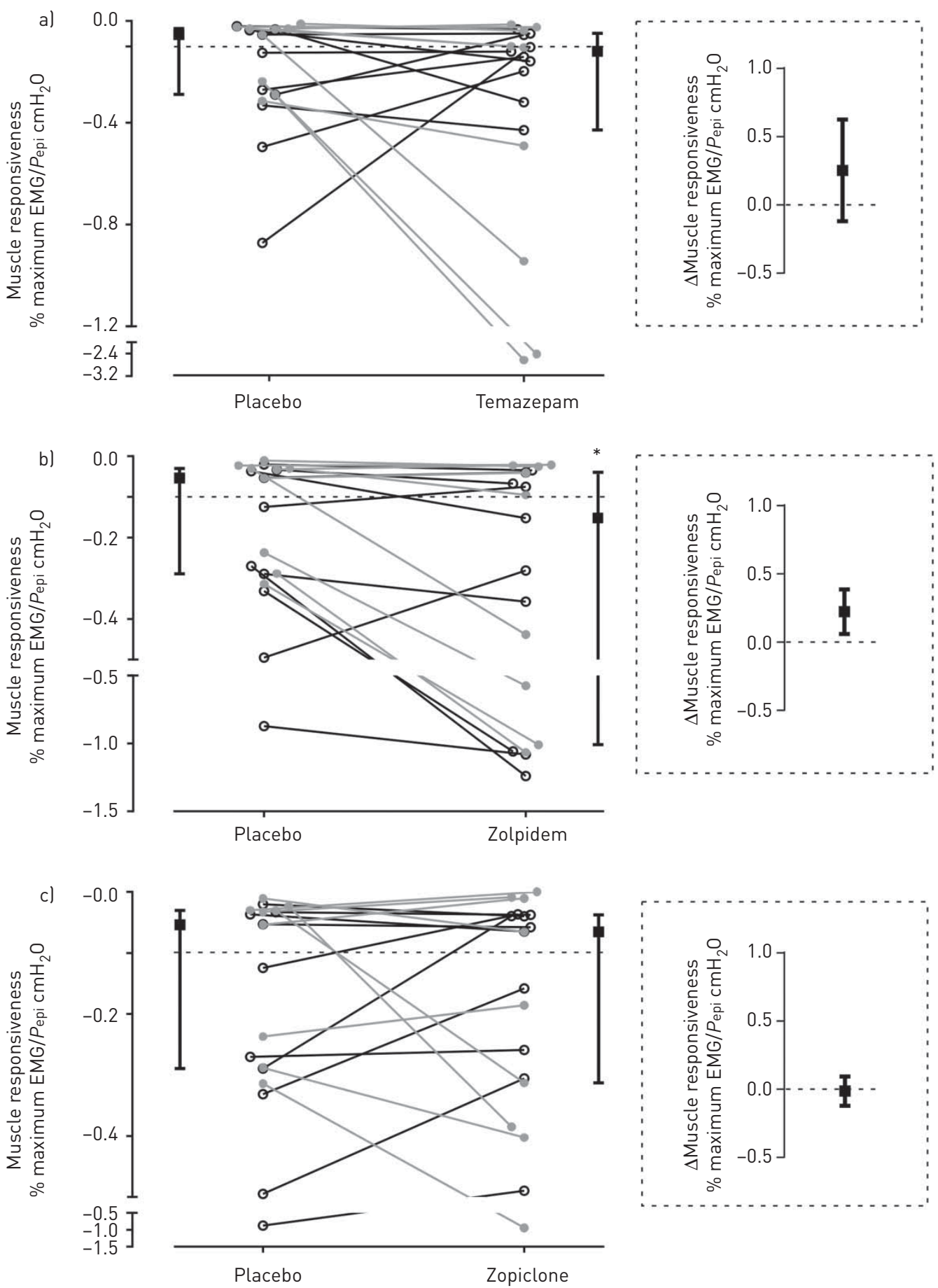

FIGURE 4 Individual muscle responsiveness (slope of peak genioglossus electromyography (EMG) versus negative epiglottic pressure $\left(P_{\text {epi }}\right)$ data for obstructive sleep apnoea (OSA) (open circles) and non-OSA participants (grey circles) during upper airway narrowing or airway occlusion following reductions in continuous positive airway pressure with a) temazepam, b) zolpidem and cl zopiclone compared to placebo. Group median and interquartile range are indicated (black squares) adjacent to each condition. Values above the dashed horizontal line represent poor muscle responsiveness as defined previously [21]. *: significant difference compared to placebo. Insets indicate the mean difference from placebo for each drug with $95 \%$ confidence intervals. Data in all four conditions were available in $\mathrm{n}=19$ participants (poor EMG signal in one individual and inadequate epiglottic pressure data in another, both during the placebo night).

\section{Benzodiazepines}

A lack of increase in arousal threshold with temazepam contrasts with studies by BERRY et al. [24] using $0.25 \mathrm{mg}$ of triazolam in healthy individuals and in people with OSA [10]. The $\sim 2 \mathrm{cmH}_{2} \mathrm{O}$ absolute change 


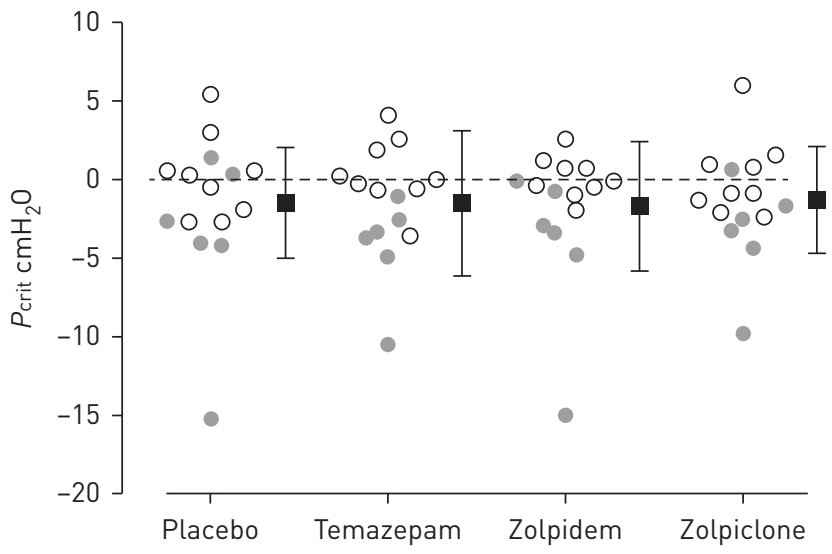

FIGURE 5 Individual upper airway collapsibility $\left(P_{\text {crit }}\right)$ data for obstructive sleep apnoea (OSA) (open circles) and non-OSA participants (grey circles) during non-REM sleep. Group mean \pm SD are indicated (black squares) adjacent to each condition. None of the hypnotics significantly changed $P_{\text {crit }}$ compared to placebo. Data in all four conditions were available in $\mathrm{n}=15$ participants linsufficient flow-limited breaths to calculate $P_{\text {crit }}$ during the placebo condition and/or additional nights in five individuals, one other individual only had $P_{\text {crit }}$ data available during the placebo night).

detected in the current study compared with $\sim 7 \mathrm{cmH}_{2} \mathrm{O}$ [24] and $\sim 9 \mathrm{cmH}_{2} \mathrm{O}$ [10] in the previous studies is much lower. The baseline arousal threshold on placebo was also lower in the current than the prior benzodiazepine studies (e.g. -15 versus $\sim-20$ and $\sim-45 \mathrm{cmH}_{2} \mathrm{O}$ respectively). Indeed, the OSA patients in the triazolam study already had impaired arousal thresholds. Thus, in addition to potential drug and potency differences (10 mg of temazepam versus $0.25 \mathrm{mg}$ of triazolam), differences in participant characteristics may have contributed to the contrasting findings.

Diazepam reduced output from hypoglossal motor neurons in decerebrate cats [25]. In contrast, $10 \mathrm{mg}$ of diazepam had no effect on peak genioglossus EMG activity awake during quiet breathing in healthy humans [5]. However, genioglossus EMG activity was reduced by $\sim 40 \%$ during $\mathrm{CO}_{2}$ rebreathing at an end-tidal carbon dioxide tension of $52 \mathrm{mmHg}$ but not $58 \mathrm{mmHg}$. Rather than a direct drug effect, sleep intrusion may have been responsible for the observed reduction in EMG with diazepam. Indeed, while EEG was not recorded, tidal volume declined in some individuals during the $52 \mathrm{mmHg}$ condition, which is consistent with sleep intrusion [5].

In contrast, the findings of the current study are the first to show that temazepam does not reduce genioglossus activity in humans during stable non-REM and REM sleep or when challenged during airway narrowing. However, among the group, there was greater variability in genioglossus muscle responsiveness during airway narrowing indicating that some individuals are indeed more prone to reductions in muscle activity with temazepam than others. Identification of the characteristics of these individuals is a priority for future research. Nonetheless, consistent with no overall systematic reductions in pharyngeal muscle activity, $P_{\text {crit }}$ was not altered by temazepam. This is similar to low-dose sedation with the benzodiazepine midazolam [26] and $100 \mathrm{mg}$ of the antidepressant trazodone [8], both of which do not alter upper airway collapsibility in people with OSA.

\section{Non-benzodiazepine hypnotics: Z drugs}

This is the first study to determine the effect of zolpidem on the respiratory arousal threshold in humans. Consistent with other $\mathrm{Z}$ drug studies of eszopiclone and zopiclone, the arousal threshold was $~ 27 \%$ higher with $7.5 \mathrm{mg}$ of zolpidem than placebo $[6,17]$. There is limited data in humans on the effects of $\mathrm{Z}$ drugs on upper airway muscle activity. Data from a small number of participants with OSA indicate that genioglossus EMG activity on stable CPAP and muscle responsiveness during respiratory events are not impaired by $10 \mathrm{mg}$ of zopiclone [6, 7]. Indeed, genioglossus EMG activity was not lower during the first and last breaths of respiratory events than the placebo [6]. Similarly, the current findings do not show any evidence for a systematic reduction in central neural drive to genioglossus during sleep when upper airway resistance and therefore reflex input, is minimised with CPAP. Interestingly, when the upper airway was challenged during narrowing and collapse, the genioglossus muscle responded three times more robustly with zolpidem than placebo. Given that genioglossus muscle activity on therapeutic CPAP which predominantly reflects central neural drive was not increased with zolpidem, increased pharyngeal muscle responsiveness to airway narrowing is consistent with enhanced reflex sensitivity. This finding was unexpected, as zolpidem (like temazepam and zopiclone) acts on GABA receptors that may be expected to 
inhibit hypoglossal motoneurons [27] as reported with zolpidem in animals [28]. However, in rats, systemic administration of zolpidem increases the arousal threshold to $\mathrm{CO}_{2}$ stimulation and genioglossus muscle activity is higher immediately prior to arousal and during baseline non-REM sleep than placebo [28]. This paradoxical excitatory motor activity produced by zolpidem in the current and previous study [28] is consistent with case reports/studies in which zolpidem improves motor function in patients with stroke, Parkinson's and neurological disorders [29-33]. Recent evidence indicates a receptor target that is unique for zolpidem [34]. However, further studies are required to determine the underlying mechanisms mediating these effects.

The use of CPAP and its role in reducing pharyngeal muscle activity needs to be considered when interpreting the magnitude of change in muscle responsiveness. Thus, it will be important to conduct future studies without CPAP to determine if the three-fold increases in muscle responsiveness with zolpidem in the current study translate to non-CPAP conditions and its effect on OSA severity. If so, this size of increase could be quite important, at least for some OSA patients. In the current experiment two-thirds of the healthy individuals and $\sim 40 \%$ of the OSA patients had poor genioglossus responsiveness $\left(<0.1 \%\right.$ increase in maximal genioglossus activity in response to a $1 \mathrm{cmH}_{2} \mathrm{O}$ increase in negative pharyngeal pressure) during sleep at baseline. This confirms previous reports that many people with and without OSA do not respond to mechanoreceptor feedback during sleep despite substantial respiratory stimuli $[7,21,35]$. This finding is also consistent with the concept that a threshold level of respiratory stimuli is required for pharyngeal muscle reflex activation $[7,36]$ which for many people is quite high and may be close to the arousal threshold [7]. Rates of poor muscle responsiveness in the non-OSA participants were higher in the current study than in our prior study [21]. While the reasons for the disparity are unclear, this finding reinforces the importance of the interaction between anatomical and non-anatomical factors in driving OSA pathogenesis [37].

\section{Methodological considerations}

This is the largest physiological study to determine the effects of common hypnotics in humans during sleep. However, there are several limitations. Surprisingly, we did not detect a significant increase in respiratory arousal threshold with temazepam. The mean change in arousal threshold of $2.3 \pm 5.1 \mathrm{cmH}_{2} \mathrm{O}$ from placebo was smaller than anticipated based on the changes detected using standard doses of other hypnotics $[6,8,9,17]$. Thus, a type 2 error for this comparison is possible. Nonetheless, a change in the arousal threshold of this magnitude is unlikely to be clinically significant. Thus, the findings of the current study suggest that any effects of $10 \mathrm{mg}$ of temazepam on the arousal threshold are less pronounced than standard doses of zolpidem and zopiclone. It is important to acknowledge, that while $10 \mathrm{mg}$ of temazepam is a standard clinical dose in Australia recommended under the National Pharmaceutical Benefit Scheme, there are regional differences in temazepam dosing practices (standard doses range between 7.5 to $30 \mathrm{mg}$ ). Thus, $10 \mathrm{mg}$ of temazepam may not have an equivalent hypnotic effect compared to the doses of $\mathrm{Z}$ drugs tested. It remains possible that a higher temazepam dose may yield similar changes in arousal threshold to the doses of $\mathrm{Z}$ drugs used here.

Another consideration is that measurements were acquired across the night and averaged to maximise signal-to-noise characteristics rather than at the point of peak concentration, which varies between agents. Nonetheless, despite differences in the half-lives between drugs, we did not detect time of night differences in arousal threshold or muscle responsiveness for any hypnotic (supplementary material). Thus, the changes that we detected appear relatively stable throughout the night although this requires further investigation.

Participants were heavily instrumented in this study, which may have affected the findings. However, recent analysis from our laboratory (unpublished) has shown that sleep efficiency, arousal index and the AHI are not different during standard polysomnography compared with a sleep physiology study performed using a nasal mask, pneumotachograph and epiglottic catheter. Given the repeated measures study design in which each individual acts as their own control, the study equipment is unlikely to have impacted our key findings. Similarly, the study was not designed to look at spontaneous occurring respiratory events, thus the effects of hypnotics on the respiratory event count, duration and oxygen saturation could not be explored.

An overly sensitive ventilatory control system, or a high loop gain can also contribute to the pathogenesis of OSA [21]. Whether benzodiazepines or $\mathrm{Z}$ drugs alter ventilatory control was not determined in this study. Previously, OSA patients with higher chemosensitivity when awake had greater respiratory depression with temazepam [38]. However, we found that all hypnotics increased breathing frequency compared to placebo albeit by a small degree. This was unexpected but is consistent with increases in breathing frequency that occur during REM sleep, a sleep stage that tends to be protected from respiratory control instability [39]. The primary comparisons were also limited to non-REM sleep in the current 
study. Thus, the effects of hypnotics on the phenotypic traits that contribute to OSA during REM sleep remain unclear.

\section{Summary and clinical implications}

These findings challenge the concept that hypnotics reduce central drive to pharyngeal and other inspiratory muscles during sleep. Thus, the hypnotics tested in the current study are unlikely to worsen OSA via a reduction in drive. Similarly, the existing evidence does not indicate that these doses of common hypnotics systematically worsen hypoxaemia or OSA severity despite increases in the threshold for arousal. This is favourable from a safety perspective [6, 8-12, 14, 17, 40,41]. Nonetheless, despite the present findings, caution remains warranted as some people with OSA do experience major reductions in oxygenation with certain hypnotics. While there is currently limited evidence, poor outcomes tend to occur more commonly at high doses [11], in people who already have high thresholds for arousal [9], and in obese individuals with very severe OSA $[6,10]$. Thus, further work is required to identify which patients are most at risk of harm and with which agents and doses.

The paradoxical increase in genioglossus muscle responsiveness with zolpidem is intriguing. Improvements in central sleep apnoea and daytime sleepiness have been observed with zolpidem [41]. These effects are likely mediated via sleep promotion and arousal prevention rather than pharyngeal dilator muscle activation. However, given the overlap in pathophysiology between central and obstructive sleep apnoea $[42,43]$, a protective role of increased genioglossus muscle responsiveness with zolpidem in central sleep apnoea cannot be dismissed. Thus, further mechanistic and clinical trials with zolpidem in people with sleep disordered breathing in the absence of CPAP are required. Indeed, the current findings suggest that zolpidem has the potential to target two common causes of OSA by increasing the threshold for arousal and increasing pharyngeal muscle activation.

\section{Acknowledgements}

The authors would like to thank Benjamin Tong and Rachel McBain for sleep staging and Janet Taylor for coordinating the randomisation. We are also grateful to Barbara Toson, Rob Herbert and Brigid Betz-Stablein for their statistical advice and to all the participants who took part in the study.

Author contributions: J.C. Carberry wrote the manuscript and all of the authors contributed to data interpretation and the final version of the manuscript. J.C. Carberry and L.P. Fisher collected and analysed the data. D.J. Eckert assisted with data collection and analysis and was responsible for the study conception and design.

\section{References}

1 Vozoris NT, Leung RS. Sedative medication use: prevalence, risk factors, and associations with body mass index using population-level data. Sleep 2011; 34: 869-874.

2 Colell E, Sanchez-Niubo A, Domingo-Salvany A, et al. Prevalence of the use of hypnotics and sedatives among the working population and associated work-related stress factors. Gac Sanit 2014; 28: 369-375.

3 Young T, Skatrud J, Peppard PE. Risk factors for obstructive sleep apnea in adults. JAMA 2004; 291 : $2013-2016$.

4 Guilleminault C. Benzodiazepines, breathing, and sleep. Am J Med 1990; 88: 25S-28S.

5 Leiter JC, Knuth SL, Krol RC, et al. The effect of diazepam on genioglossal muscle activity in normal human subjects. Am Rev Respir Dis 1985; 132: 216-219.

6 Carter SG, Berger MS, Carberry JC, et al. Zopiclone increases the arousal threshold without impairing genioglossus activity in obstructive sleep Apnea. Sleep 2016; 39: 757-766.

7 Loewen $\mathrm{AH}$, Ostrowski M, Laprairie J, et al. Response of genioglossus muscle to increasing chemical drive in sleeping obstructive apnea patients. Sleep 2011; 34: 1061-1073.

8 Eckert DJ, Malhotra A, Wellman A, et al. Trazodone increases the respiratory arousal threshold in patients with obstructive sleep apnea and a low arousal threshold. Sleep 2014; 37: 811-819.

9 Eckert DJ, Younes MK. Arousal from sleep: implications for obstructive sleep apnea pathogenesis and treatment. J Appl Physiol 2014; 116: 302-313.

10 Berry RB, Kouchi K, Bower J, et al. Triazolam in patients with obstructive sleep apnea. Am J Respir Crit Care Med 1995; 151: 450-454.

11 Cirignotta F, Mondini S, Zucconi M, et al. Zolpidem-polysomnographic study of the effect of a new hypnotic drug in sleep apnea syndrome. Pharmacol Biochem Behav 1988; 29: 807-809.

12 Hoijer U, Hedner J, Ejnell H, et al. Nitrazepam in patients with sleep apnoea: a double-blind placebo-controlled study. Eur Respir J 1994; 7: 2011-2015.

13 Camacho ME, Morin CM. The effect of temazepam on respiration in elderly insomniacs with mild sleep apnea. Sleep 1995; 18: 644-645.

14 Quera-Salva MA, McCann C, Boudet J, et al. Effects of zolpidem on sleep architecture, night time ventilation, daytime vigilance and performance in heavy snorers. Br J Clin Pharmacol 1994; 37: 539-543.

15 Younes M. Role of arousals in the pathogenesis of obstructive sleep apnea. Am J Respir Crit Care Med 2004; 169: 623-633.

16 Smales ET, Edwards BA, Deyoung PN, et al. Trazodone effects on obstructive sleep apnea and non-REM arousal threshold. Ann Am Thorac Soc 2015; 12: 758-764.

17 Eckert DJ, Owens RL, Kehlmann GB, et al. Eszopiclone increases the respiratory arousal threshold and lowers the apnoea/hypopnoea index in obstructive sleep apnoea patients with a low arousal threshold. Clin Sci (Lond) 2011; 120: 505-514. 
18 Edwards BA, Eckert DJ, McSharry DG, et al. Clinical predictors of the respiratory arousal threshold in patients with obstructive sleep apnea. Am J Respir Crit Care Med 2014; 190: 1293-1300.

19 Gray EL, McKenzie DK, Eckert DJ. Obstructive sleep apnea without obesity is common and difficult to treat: evidence for a distinct pathophysiological phenotype. J Clin Sleep Med 2017; 13: 81-88.

20 Berry RB, Budhiraja R, Gottlieb DJ, et al. Rules for scoring respiratory events in sleep: update of the 2007 AASM Manual for the Scoring of Sleep and Associated Events. Deliberations of the Sleep Apnea Definitions Task Force of the American Academy of Sleep Medicine. J Clin Sleep Med 2012; 8: 597-619.

21 Eckert DJ, White DP, Jordan AS, et al. Defining phenotypic causes of obstructive sleep apnea. Identification of novel therapeutic targets. Am J Respir Crit Care Med 2013; 188: 996-1004.

22 Carberry JC, Hensen H, Fisher LP, et al. Mechanisms contributing to the response of upper-airway muscles to changes in airway pressure. J Appl Physiol 2015; 118: 1221-1228.

23 Ludbrook J. Multiple comparison procedures updated. Clin Exp Pharmacol Physiol 1998; 25: 1032-1037.

24 Berry RB, McCasland CR, Light RW. The effect of triazolam on the arousal response to airway occlusion during sleep in normal subjects. Am Rev Respir Dis 1992; 146: 1256-1260.

25 Bonora M, St John WM, Bledsoe TA. Differential elevation by protriptyline and depression by diazepam of upper airway respiratory motor activity. Am Rev Respir Dis 1985; 131: 41-45.

26 Genta PR, Eckert DJ, Gregorio MG, et al. Critical closing pressure during midazolam-induced sleep. J Appl Physiol 2011; 111: 1315-1322.

27 Morrison JL, Sood S, Liu H, et al. GABAA receptor antagonism at the hypoglossal motor nucleus increases genioglossus muscle activity in NREM but not REM sleep. J Physiol 2003; 548: 569-583.

28 Park E, Younes M, Liu H, et al. Systemic vs. central administration of common hypnotics reveals opposing effects on genioglossus muscle activity in rats. Sleep 2008; 31: 355-365.

29 Cohen SI, Duong TT. Increased arousal in a patient with anoxic brain injury after administration of zolpidem. Am J Phys Med Rehabil 2008; 87: 229-231.

30 Chen YY, Sy HN, Wu SL. Zolpidem improves akinesia, dystonia and dyskinesia in advanced Parkinson's disease. J Clin Neurosci 2008; 15: 955-956.

31 Chang AY, Weirich E. Trial of zolpidem, eszopiclone, and other GABA agonists in a patient with progressive supranuclear palsy. Case Rep Med 2014; 2014: 107064.

32 Daniele A, Moro E, Bentivoglio AR. Zolpidem in progressive supranuclear palsy. N Engl J Med 1999; 341: 543-544.

33 Bomalaski MN, Claflin ES, Townsend W, et al. Zolpidem for the treatment of neurologic disorders: a systematic review. JAMA Neurology 2017; 74: 1130-1139.

34 Che Has AT, Absalom N, van Nieuwenhuijzen PS, et al. Zolpidem is a potent stoichiometry-selective modulator of alpha1beta3 GABAA receptors: evidence of a novel benzodiazepine site in the alpha1-alpha1 interface. Sci Rep 2016; 6: 28674

35 Jordan AS, Wellman A, Heinzer RC, et al. Mechanisms used to restore ventilation after partial upper airway collapse during sleep in humans. Thorax 2007; 62: 861-867.

36 Martins RT, Carberry JC, Eckert DJ. Breath-to-breath reflex modulation of genioglossus muscle activity in obstructive sleep apnea. Sleep Med 2016; 21: 45-46.

37 Eckert DJ. Phenotypic approaches to obstructive sleep apnoea - new pathways for targeted therapy. Sleep Med Rev 2016; in press [https://doi.org/10.1016/j.smrv.2016.12.003].

38 Wang D, Marshall NS, Duffin J, et al. Phenotyping interindividual variability in obstructive sleep apnoea response to temazepam using ventilatory chemoreflexes during wakefulness. J Sleep Res 2011; 20: 526-532.

39 Eckert DJ, Jordan AS, Merchia P, et al. Central sleep apnea: pathophysiology and treatment. Chest 2007; 131: 595-607.

40 Murciano D, Armengaud $\mathrm{MH}$, Cramer $\mathrm{PH}$, et al. Acute effects of zolpidem, triazolam and flunitrazepam on arterial blood gases and control of breathing in severe COPD. Eur Respir J 1993; 6: 625-629.

41 Quadri S, Drake C, Hudgel DW. Improvement of idiopathic central sleep apnea with zolpidem. J Clin Sleep Med 2009; 5: 122-129.

42 Eckert DJ, Malhotra A, Jordan AS. Mechanisms of apnea. Prog Cardiovasc Dis 2009; 51: 313-323.

43 Sankri-Tarbichi AG, Rowley JA, Badr MS. Expiratory pharyngeal narrowing during central hypocapnic hypopnea. Am J Respir Crit Care Med 2009; 179: 313-319. 